\title{
X-ray observations of massive colliding wind binaries
}

\author{
Michael F. Corcoran \\ Universities Space Research Association, \\ 7501 Forbes Blvd, Ste 206, Seabrook, MD20706, USA, \\ and \\ Laboratory for High Energy Astrophysics, \\ NASA Goddard Space Flight Center, Greenbelt, MD 20771, USA
}

\begin{abstract}
Massive stars in binary systems can generate X-ray emission in the region between the two stars where stellar winds collide. Colliding wind $\mathrm{X}$-ray emission acts as an in-situ probe of important wind parameters like massloss rates, chemical abundances, wind velocities, and possibly magnetic field strengths. Variations in observed colliding-wind X-ray emission can be produced by the changing line-of-sight to the colliding wind region as the stars move in orbit and/or the changing emission measure of the shocked gas in the wind collision zone produced if the separations between the two stars change. X-ray variability depends on the wind and orbital parameters and so can in principal allow the direct measurement of mass functions even for low inclination systems and provide a connection between the stellar and wind parameters. X-ray emission from colliding wind systems can thus be used to refine our understanding of the evolutionary state of massive stars and to help test evolutionary models. We discuss recent advances in defining the X-ray spectral and temporal signatures of colliding wind X-ray emission using ROSAT, ASCA, RXTE, Chandra and XMM-Newton data.
\end{abstract}

\section{Introduction}

Cherepashchuk (1976) and Prilutskii \& Usov (1976) first considered the production of X-radiation by massive, non-degenerate binary systems due to the collision of the wind from one star with the wind or surface of the companion. In such cases a bow shock forms and the wind kinetic energy is converted into thermal energy at the shock front producing temperatures of millions of degrees and thermal Bremsstrahlung X-rays and line emission with luminosities in the $0.2-10 \mathrm{keV}$ band of $L_{\mathrm{x}} \simeq 10^{32-34} \mathrm{erg} \mathrm{s}^{-1}$. Prior to the launch of the Einstein $\mathrm{X}$-ray observatory in 1978, single massive stars were not expected to be X-ray sources (because they were thought to lack the outer convective envelopes necessary for the familiar dynamo effect), so any mechanism by which massive stars could produce X-ray emission was of special interest. In particular, detection of colliding wind X-ray emission could be a useful tool for the identification of Wolf-Rayet binaries, as Cherepashchuk originally noted. Since colliding wind $\mathrm{X}$-ray emission is in theory observable regardless of orbital inclination, observations of colliding wind X-rays provide an important complement to standard photometric and spectrometric means of binary star identification and analy- 
sis. Because colliding wind X-ray emission depends on the orbital separation as a function of time, and also on the wind parameters (mass loss rates, wind speeds) of the two stars, and because the amount of X-ray absorption depends on the wind density of the frontmost star, colliding wind X-ray spectra provide an extremely useful probe of the physical parameters of massive binaries and an in-situ probe of the radiatively driven winds.

With the detection of shock heated X-ray emitting gas in the winds of apparently single OB and WR stars, the mere detection of X-ray emission was no longer sufficient evidence of a massive star's duplicity. However, since emission from single stars is apparently dominated by low-temperature emission which is not highly variable, detection of hard, variable emission from a massive star often implies the presence of a wind-wind collision. Thus hard X-ray monitoring observations complement more familiar photometric and spectrometric methods which are generally used to identify massive binaries.

In this paper, we briefly review the production and observed properties of X-rays from colliding winds in massive binaries. In general X-ray emission from non-explosive colliding massive outflows is important in a wide range of astrophysical processes, such as the discovery of X-ray emission from the interactions of WR and AGB-phase winds in planetary nebulae (Kastner et al. 2000; Chu et al. 2001), the interactions of photoionized wakes with stellar winds in high mass X-ray binaries (Blondin, Stevens \& Kallman 1991), the interactions of winds from one or more massive stars and the local ISM to produce diffuse X-ray emission (Yusef-Zadeh et al. 2002), and, on larger scales the interaction of galactic 'superwinds' from starbursts with halo gas (Suchkov et al. 1994; Strickland et al. 2002). Due to space limitations we can only mention in passing these interesting cases,

\section{Colliding wind X-ray emission}

The interaction of a large-scale outflow (or mass ejection) from a star with another stellar outflow (or with a nearby cloud, or with the interstellar medium) can produce a strong shock with temperatures in the shocked gas related to the perpendicular flow velocity $v_{\perp}$ as $T \approx 3 \times 10^{7}\left(v_{\perp} / 1000 \mathrm{~km} \mathrm{~s}^{-1}\right)^{2} \mathrm{~K}$ (Prilutskii \& Usov 1976), if the mean molecular weight of the gas $\mu=4 / 3$ and the adiabatic index $\gamma=5 / 3$. For typical wind speeds $>1000 \mathrm{~km} \mathrm{~s}^{-1}$, the temperature in the shocked gas should be $>3 \times 10^{7} \mathrm{~K} \approx 2.5 \mathrm{keV}$. If the mass loss rates from each binary component are $\dot{M}_{1}, \dot{M}_{2}$ with wind terminal velocities $v_{\infty, 1}, v_{\infty, 2}$ respectively, and the stellar component separation is $D$, then the distance from the first star to the shock front is $r_{1}=D \times \eta /(1+\eta)$, where $\eta=\left(\dot{M}_{1} v_{\infty, 1} / \dot{M}_{2} v_{\infty, 2}\right)^{-1}$ and the distance from the second star to the shock front $r_{2}=D-r_{1}$ by equating dynamic pressures. The shock cone is concave towards the star whose wind momentum flux $\dot{M} v_{\infty}$ is lower. In general the shape of the shock will be altered by the orbital motions of the stars. The observed X-ray flux will depend on orbital phase due to occultation of the shock front if the orbital inclination is large enough, while the intrinsic flux from the shock will vary with phase due to changes in the stellar separation in eccentric systems and/or due to the onset of sudden radiative braking, in which the photon flux from one star decelerates the wind from the other (Owocki \& Gayley 1995). 


\section{Observed properties of colliding wind X-ray emission from non- compact, massive binaries}

Attempts in the 1970's and 1980's (Cooke, Fabian \& Pringle 1978; Bradt \& Kelley 1979; Moffat et al. 1982; Pollock 1987) to prove the existence of colliding wind $\mathrm{X}$-ray emission from OB and WR binaries met with little success, due to four reasons: early X-ray detectors were not very sensitive; there was little temporal coverage; the fields are crowded; and $\mathrm{X}$-ray emission from radiative instabilities in individual stellar winds (at a level $L_{\mathrm{x}} / L_{\mathrm{bol}} \approx 10^{-7}$, Chlebowski et al. 1989 ; Berghöfer et al. 1997) contaminated the colliding wind signal. However, surveys with the Einstein observatory showed that, in general, binary OB and WR stars tended to be brighter than single stars (Pollock 1987; Chlebowski \& Garmany 1991), but with large scatter. EXOSAT observations of WR 140 (= HD 193793, WC7+O4-5) in the 1980's provided the best early case of colliding wind emission in a massive binary (Williams et al. 1990). These observations revealed a large X-ray overluminousity $\left(L_{\mathrm{x}} / L_{\mathrm{bol}} \simeq 10^{-5}\right)$, which suggested enhanced emission perhaps from a wind-wind collision. This suggestion was confirmed by the discovery of a periodic IR outburst and the star was recognized as a long period $(P=7.94 \mathrm{yr})$, eccentric $(e=0.85)$ binary system (Williams et al. $1987 ; 1990)$.

ROSAT made the first substantial strides in providing evidence of colliding wind X-ray emission in a significant number of important massive binaries by detecting phase-locked X-ray variability. While factors of two variation in Xray emission might be expected even in the absence of colliding wind emission (if each member star possesses similar intrinsic X-ray luminosities), ROSAT observations of stars like $29 \mathrm{CMa}, \gamma^{2}$ Vel, Plaskett's Star, WR 140, and others showed stronger X-ray variations. Often the X-ray emission reached maximum intensity when the star possessing the less-dense wind was in front, apparently due to a decrease in absorption to the colliding wind shock.

Spectra obtained by the ASCA X-ray observatory show that massive binaries are typically hotter than single stars. Colliding wind spectra is often a mix of temperatures and shows strong line emission from $\mathrm{H}$-like and He-like ions of $\mathrm{Mg}, \mathrm{Si}, \mathrm{S}$, and $\mathrm{Fe}$, with additional lines being discovered more recently in high resolution X-ray grating observations with Chandra and XMM-Newton.

Below we present a limited survey of colliding-wind X-ray emission of three WR binaries and the peculiar star $\eta$ Car. WR catalogues numbers, spectral types and binary parameters are from the catalogue by van der Hucht (2001).

\subsection{V444 Cygni (WR 139, WN5+O6 III-V, $P=4.21 \mathrm{~d}, e=0.04$ )}

Colliding wind effects in V444 Cygni have been well documented in the UV and optical (Shore \& Brown 1988; Marchenko et al. 1997; Flores et al. 2001). It was expected that V444 Cyg would exhibit colliding wind X-ray emission at a level $L_{\mathrm{x}} \approx 10^{34-35} \mathrm{erg} \mathrm{s}^{-1}$ (Cherepashchuk 1976; Stevens, Blondin \& Pollock 1992), but in fact V444 Cyg appears as a rather weak X-ray source. Moffat et al. (1982) and Pollock (1987) presented Einstein observations over four consecutive days, which showed a variation in the $0.4-4.5 \mathrm{keV}$ band of about a factor of two, with flux minimum at phase $\phi=0$ (when the WN5 star is in front of the O6 star). ROSAT (Corcoran et al. 1996) saw essentially the same X-ray behavior, with $4 \times 10^{32}<L_{\mathrm{x}}<8 \times 10^{32} \mathrm{ergs} \mathrm{s}^{-1}$ from inside to outside eclipse. This is not much larger than the expected X-ray luminosity of the O6 star, $\sim 6 \times 10^{32} \mathrm{erg} \mathrm{s}^{-1}$, so 
that the contribution of colliding wind emission to the observed flux is rather minimal. Probably the best evidence of colliding wind emission in this source is the variable hard X-ray component detected in three observations with $A S C A$ (Maeda et al. 1999). The column density and the absorption-corrected flux both reached maximum when the WN5 star was in front, while the observed flux reached a maximum at quadrature.

As noted by Owocki \& Gayley (1995), the onset of 'sudden radiative breaking' (where the UV flux from the O-type component decelerates the wind from the WR star and weakens the shock) might account for the relatively weak X-ray emission from the colliding wind shock in this system.

\section{2. $\gamma^{2}$ Velorum (WR 11, WC8+O7.5 III-V, $P=78.53 \mathrm{~d}, e=0.33$ )}

$\gamma^{2}$ Vel is a moderate period eccentric $(e=0.33)$ binary. The distance to the star has been accurately measured by Hipparchos as $0.26 \mathrm{kpc}$ (van der Hucht et al. 1997), and the mass loss rates are $2.8 \times 10^{-5}$ and $1.8 \times 10^{-7} \mathrm{M}_{\odot} \mathrm{yr}^{-1}$ from the WC8 and O7.5III star, respectively (De Marco \& Schmutz 1999). Colliding wind X-ray emission was convincingly demonstrated by Willis, Schild \& Stevens (1995) who showed that the X-ray flux reaches a maximum near $\phi=0.5$, the time when the O-type component was in front (which occurs near periastron). Moderate resolution $0.5-10 \mathrm{keV}$ spectra were obtained by the ASCA observatory at $\phi=0.4,0.5$ (Stevens et al. 1996) and 0.0 (WC8 star in front, Rauw et al. 2000). A high-resolution grating spectrum was recently obtained with the Chandra-HETGS instrument, just after periastron (Skinner et al. 2001). These grating observations show that the emission is multi-temperature (though some contamination from nearby sources is expected) and that the flux from the hot component increases by more than a factor of four and suffers variable absorption. The spectral variation is consistent with the expectations of colliding wind models, but the intrinsic luminosity doesn't seem to follow the $1 / D$ law very well; however there is substantial uncertainty in correcting the observed luminosity to derive the intrinsic luminosity, so some of this discrepancy may be due to systematic errors in determining the absorbing column. Also the X-ray maximum occurs a bit after the passage of the $\mathrm{O}$ star, which perhaps is evidence of some skewness to the shock cone due to orbital motion. Stevens et al. modelled the ASCA spectrum using a synthetic colliding wind model spectrum and derived a mass loss rate for the WC8 star of $3 \times 10^{-5} \mathrm{M}_{\odot} \mathrm{yr}^{-1}$, in good agreement with the later determination by De Marco \& Schmutz (1999), but which was a factor of three lower than the mas loss rate derived from free-free emission in the radio. However, the later revision (downward) of the distance to the star by Hipparchos reduced the radio mass-loss rate and brought the $\mathrm{X}$-ray and radio rates into agreement. The X-ray emission lines seen in the grating spectra are broad but unshifted, and the He-like lines show strong forbidden emission, suggesting an origin far from the surface of either star. This is in contrast with the He-like lines in the X-ray grating spectra of $\zeta$ Pup (Kahn et al. 2001) and $\zeta$ Ori (Waldron \& Cassinelli 2001), neither of which have strong forbidden emission lines.

\subsection{WR 140 (HD 193793, WC7+O4-5, $P=2900 \mathrm{~d}, e=0.85$ )}

After discovery of its X-ray 'overluminosity' by EXOSAT, and its UV, IR, and radio periodicity (Williams et al. 1987, 1990, 1994; Setia Gunawan et al. 2001), 
X-ray emission from WR 140 was monitored by the ROSAT and ASCA satellites at select phases around the orbit, and most recently by $R X T E$ around the time of the last periastron passage in March, 2001. Near periastron the flux rises quickly as the stellar separation decreases, but as the WR star moves in front of the O-type component, the colliding wind shock is occulted by the WR star, causing a rapid, brief $\mathrm{X}$-ray minimum. The shoulders of the $\mathrm{X}$-ray minimum are asymmetric in that the observed flux is higher before periastron than after egress from the minimum, due to increased residual absorption from the WR star's wind after periastron passage. The derived column density $N_{\mathrm{H}}$ clearly reaches a maximum near periastron passage. Naively correcting the RXTE spectrum near periastron for the observed value of $N_{\mathrm{H}}$ does not produce a maximum in the intrinsic flux curve, though the colliding wind model requires that the intrinsic X-ray flux from the shock be maximum at periastron. This may be an observational effect (i.e., that the spectrum measured by $R X T E$ at periastron is really the 'sky' background), or it might be due to a real physical effect like the onset of sudden radiative braking.

Pollock et al. (in preparation) have obtained a set of grating spectra with the Chandra-HETGS around the time of X-ray maximum, and during the recovery from minimum. Like $\gamma^{2}$ Vel, the X-ray spectrum near maximum shows multitemperature thermal emission with strong He-like and $\mathrm{H}$-like lines from $\mathrm{NeX}$, Mg XI, MgxiI, Si XIII, Si XIV, Sxv, Sxvi, Caxix and Fexxv. The Nex line is particularly strong - a simple 2-temperature Mewe-Kaastra-Liedahl collisional plasma fit suggests a $60 \times$ overabundance of $\mathrm{Ne}$, with $\mathrm{k} T_{1}=4.8 \mathrm{keV}$ and $\mathrm{k} T_{2}=0.9$ (corresponding to pre-shock velocities of $\sim 1400 \mathrm{~km} \mathrm{~s}^{-1}$ and $600 \mathrm{~km} \mathrm{~s}^{-1}$, respectively). Near X-ray maximum the emission lines are broad and blue-shifted by about $-600 \mathrm{~km} \mathrm{~s}^{-1}$. The line-broadening does not appear constant in the preperiastron spectrum but declines with wavelength from 2000 to less than 1000 $\mathrm{km} \mathrm{s}^{-1}$. In the spectrum after X-ray recovery, the lines are broader and slightly redshifted.

\section{4. $\quad \eta$ Carinae (pec., $P=2010 \mathrm{~d}$ )}

$\eta$ Car is a controversial case; it was widely regarded as a single star until the discovery of a 5.52-yr period in the IR (Whitelock et al. 1994; Feast, Whitelock \& Marang 2001) and in the equivalent width of the He I $10830 \AA$ line (Damineli, Conti \& Lopes 1997). Its X-ray properties are very similar to those of WR 140 and $\gamma^{2}$ Vel: $(i)$ the emission is hard (a few $\mathrm{keV}$ ); (ii) the emission is thermal with a mix of $\mathrm{H} \& \mathrm{He}$-like lines showing a multi-temperature distribution; and (iii) the emission is variable and shows a minimum near the time when the star with the denser wind is expected to be in front of the shocked gas. ROSAT discovered the variability of the hard emission (Corcoran et al. 1995) while the $\mathrm{X}$-ray light curve has now been studied in great detail with $R X T E$ (Corcoran et al. 1997; Ishibashi et al. 1999; Corcoran et al. 2001). The variation in 2$10 \mathrm{keV}$ X-ray brightness near X-ray minimum (thought to be near periastron passage) shows a rapid increase to maximum, a rapid decline to minimum, and recovery to a level lower than the maximum in both cases. The X-ray eclipse of $\eta$ Car lasts longer than the eclipse of WR 140. Corcoran et al. (2001) used the analytic colliding wind X-ray model of Usov (1992) to fit the RXTE X-ray light curve, and suggested a model of the system with component masses of 80 and $30 \mathrm{M}_{\odot}$ and mass loss rates of $10^{-4}$ and $10^{-5} \mathrm{M}_{\odot} \mathrm{yr}^{-1}$, with wind terminal 
speeds of 500 and $2000 \mathrm{kms}^{-1}$. To explain the difference in X-ray flux between eclipse ingress and egress and the 3-month duration of the X-ray minimum, they suggested an enhancement in the mass loss rate from the primary near periastron, though Pittard \& Corcoran (2002) suggested that this asymmetry might also be evidence of a skewed shock cone, while Ishibashi (2001) examined the role of orbital geometry in reproducing the asymmetry.

$A S C A$ provided the first spatially-resolved measure of the X-ray spectrum up to $10 \mathrm{keV}$ (Tsuboi et al. 1997; Corcoran et al. 1998; Corcoran et al. 2001), while a recent Chandra-HETGS observation (near $\phi=0.6$ in the 5.52-yr cycle) provided the first high resolution X-ray spectrum. The Chandra grating spectrum showed a mix of $\mathrm{H}$-like and He-like lines proving for the first time that the emission is non-isothermal; unlike the grating spectra of WR 140, the line profiles are narrow and unshifted. The iron fluorescence feature discovered by $A S C A$ was clearly resolved by Chandra, though the line appears to have weakened significantly, suggestive of an overall decline in the amount of scattering material.

\section{Colliding wind cluster binaries}

Observations with ROSAT, ASCA, Chandra, and XMM-Newton of OB clusters and associations, star forming regions, and $H$ II regions have identified $O B$ and WR member stars with extraordinarily hard and luminous X-ray emission. In some cases these stars are found at the center of the cluster. As an example, Rho et al. (2001) used ASCA imaging spectrography to show that HD 164492 (O7), the star which ionizes the Trifid Nebula has an $L_{\mathbf{x}} / L_{\text {bol }}>10^{-5}$ and very hard emission. This may be a colliding wind system, or there may be some contribution to the hard X-ray emission from unresolved pre-main sequence stars.

Another example is presented by Portegies Zwart et al. (2002), who argue for a dozen colliding-wind X-ray binaries in the star cluster R 136 in the 30 Doradus region.

\section{Conclusions}

$\mathrm{X}$-ray emission is a valuable probe of wind and orbital parameters of massive binaries. Recent observations of colliding wind X-ray emitting binaries have shown: $(i)$ an unexpectedly large emitting region in $\gamma^{2}$ Vel (with the Ne IX line forming at $r>2 a$ ); (ii) an X-ray minimum due to absorption in WR 140; and (iii) unexpectedly low mass loss rates in $\eta$ Car. High spatial and spectral resolution provided by Chandra \& XMM-Newton provide a great deal of heretofore unavailable information concerning line shapes and abundances, and provide unique information about the dynamics and chemistry of the shocked gas. These observations, combined with detailed numerical models of the wind shock in two and three dimensions, should greatly expand our understanding of colliding wind systems, the connection between the stellar and wind parameters, and the importance of duplicity on stellar evolution for massive systems.

\section{References}

Berghöfer, T.W., Schmitt, J.H.M.M., Danner, R., Cassinelli, J.P. 1997, A\&A 322, 167 
Blondin, J.M., Stevens, I.R., Kallman, T.R. 1991, ApJ 371, 684

Bradt, H.V., Kelley, R.L. 1979, ApJ (Letters) 228, L33

Cherepashchuk, A.M. 1976, Pis'ma Astron. Zh. 2, 356 (= Soviet Astron. Letters 2, 138)

Chlebowski, T., Harnden, F.R., Sciortino, S. 1989, ApJ 341, 427

Chlebowski, T., Garmany, C.D. 1991, ApJ 368, 241

Chu, Y.-H., Guerrero, M.A., Gruendl, R.A., et al. 2001, ApJ (Letters) 553, L69

Cooke, B.A., Fabian, A.C., Pringle, J.E. 1978, Nature 273, 645

Corcoran, M.F., Rawley, G.L., Swank, J.H., Petre, R. 1995, ApJ 562, 1031

Corcoran, M.F., Stevens, I.R., Pollock, A.M.T., et al. 1996, ApJ 464, 434

Corcoran, M.F., Ishibashi, K., Davidson, K., et al. 1997, Nature 390, 587

Corcoran, M.F., Petre, R., Swank, J.H., et al. 1998, ApJ 494, 381

Corcoran, M.F., Petre, R., Swank, J.H., et al. 2000, ApJ 545, 420

Corcoran, M.F., Swank, J.H., Petre, R., et al. 2001, ApJ 562, 1031

Damineli, A., Conti, P.S., Lopes, D.F. 1997, New Astron. Reviews 2, 107

De Marco, O., Schmutz, W. 1999, A\&A 345,163

Feast, M., Whitelock, P., Marang, F. 2001, MNRAS 322, 741

Flores, A., Auer, L.H., Koenigsberger, G., Cardona, O. 2001, ApJ 563, 341

van der Hucht, K.A., Schrijver, H., Stenholm, B., et al. 1997, New Astron. 2, 245

van der Hucht, K.A. 2001, New Astron. Reviews 45, 135

Ishibashi, K., Corcoran, M.F., Davidson, K., Swank, J.H., et al. 1999, ApJ 524, 983

Ishibashi, K. 2001, in: T.R. Gull, S. Johansson \& K. Davidson (eds.), Eta Carinae and Other Mysterious Stars: The Hidden Opportunities of Emission Line Spectroscopy, ASP-CS 242, 53

Kahn, S.M., Leutenegger, M.A., Cottam, J., et al. 2001, A\&A (Letters) 365, 312

Kastner, J.H., Soker, N., Vrtilek, S.D., Dgani, R. 2000, ApJ (Letters) 545, L57

Maeda, Y., Koyama, K., Yokogawa, J., Skinner, S. 1999, ApJ 510, 967

Marchenko, S.V., Moffat, A.F.J., Eenens, P.R.J., et al. 1997, ApJ 485, 826

Moffat, A.F.J., Firmani, C., McLean, I.S., Seggewiss, W. 1982, in: C.W.H. de Loore \& A.J.Willis (eds.), Wolf-Rayet Stars: Observations, Physics, Evolution, Proc.

IAU Symp. No 99 (Dordrecht: Kluwer), p. 577

Owocki, S.P., Gayley, K.G. 1995, ApJ (Letters) 454, L145

Pittard, J.M., Corcoran, M.F. 2002, A\&A 383, 636

Pollock, A.M.T. 1987, ApJ 320, 283

Portegies Zwart, S.F., Pooley, D., Lewin, W.H.G. 2002, ApJ 574, 762

Prilutskii, O.F. Usov, V.V. 1976, Astron. Zh. 53, 6 (= Soviet Astron. 20, 2)

Rauw, G., Stevens, I.R., Pittard, J.M., Corcoran, M.F. 2000, MNRAS 316, 129

Rho, J., Corcoran, M.F., Chu, Y.-H., Reach, W.T. 2001, ApJ 562, 446

Setia Gunawan, D.Y.A., van der Hucht, K.A., Williams, P.M., et al. 2001, A\&A 376, 460

Shore, S.N., Brown, D.N. 1988, ApJ 334, 1021

Skinner, S.L., Güdel, M., Schmutz, W., Stevens, I.R. 2001, ApJ (Letters) 558, L113

Stevens, I.R., Blondin, J.M., Pollock, A.M.T. 1992, ApJ 386, 265

Stevens, I.R., Corcoran, M.F., Willis, A.J., et al. 1996, MNRAS 283, 589

Strickland, D.K., Heckman, T.M., Weaver, K.H., et al. 2002, ApJ 568, 689

Suchkov, A.A., Balsara, D.S., Heckman, T.M., Leitherer, C. 1994, ApJ 430, 511

Tsuboi, Y., Koyama, K., Sakano, M., Petre, R. 1997, PASJ 49, 85 
Usov, V.V. 1992, ApJ 389, 635

Waldron, W.L., Cassinelli, J.P. 2001, ApJ (Letters) 548, L45

Whitelock, P.A., Feast, M.W., Koen, C., et al. 1994, MNRAS 270, 364

Williams, P.M., van der Hucht, K.A., van der Woerd, H., et al. 1987, in: H. Lamers \&

C.W.H. de Loore (eds.), Instabilities in Luminous Early Type Stars (Dordrecht: Reidel), p. 221

Williams, P.M., van der Hucht, K.A., Pollock, A.M.T., et al. 1990, MNRAS 243, 662

Williams, P.M., van der Hucht, K.A., Spoelstra, T.A.Th. 1994, A\&A 291, 805

Willis, A.J., Schild, H., Stevens, I.R. 1995, A\&A 298, 549

Yusef-Zadeh, F., Law, C., Wardle, M., Wang, Q.D., et al. 2002, ApJ 570, 665

\section{Discussion}

CherepashchuK: For detection of clumping structure of WR wind, it is very important to detect variability of $\mathrm{X}$-ray emission from colliding wind at short time scale, less than 1 hour. Is it possible to detect such a rapid X-ray variability for WR+O binaries using modern X-ray satellites, like Chandra?

CoRCORAN: It's possible, but XMM-Newton is probably a better observatory to use, since bright sources suffer from pile-up in the Chandra CCDs which reduces any real variability. XMM-Newton doesn't suffer much from pile-up and its larger effective area means that you can examine a somewhat wider selection of sources.

KOENIGSBerger: Does the X-ray maximum in WR 140 coincide precisely with periastron passage?

Corcoran: I think that is the case; it's a bit hard to tell without modeling the X-ray light curve in detail along with the spectroscopic, radio and IR data, something we haven't done yet.

WALBORN: The X-ray nebula associated with HD 5980 is much larger than the $\eta$ Carejected nebulosities. There is spectroscopic evidence for an SNR in the vicinity of HD 5980.

CoRCoran: That's true, but I was actually referring to the large $(\sim 0.5)$ diffuse X-ray emission around $\eta$ Car. I realize it is 'mixing apples and oranges' but it is interesting. I like to note that, if you moved $\eta$ Car and it's diffuse X-ray nebula to the SMC, it would look very similar to HD 5980 and its X-ray nebula.

Koenigsberger: Pablo Velázquez has been modeling a scenario in which the SNR on the foreground of HD 5980 is interacting with the wind of HD 5980, to explain why HD 5980 is centered on the X-ray emitting region.

CorCoran: That's a great idea!

HuMPhREYS: A comment: in the Damineli et al. orbit, the 'spectroscopic event' occurred about three months before periastion passage. In the 'orbit' by Ishibashi (1999, 2001) based only on the X-ray light curve, the orbit is rotated $90^{\circ}$ and periastron occurs before the event. A second comment: HST-STIS spectra at the time of the previous event did not show an eclipse. Do you have any suggestions about the recent large flare? This does not look like the 85-d flaring seen in the previous cycle. The star itself has been brightening since 1998 . 
CoRCORAN: All the X-ray light curve modeling we've done (including Corcoran, Ishibashi, et al. 2000) shows that periastron must have occurred near late December 1997, not in March 1998 as suggested by Damineli's analysis of the radial velocity curve. Pittard et al. (1998) also assumed periastron passage near the X-ray maximum and produced a light curve, which looked remarkably like the real thing (at least up to the X-ray minimum). About the lack of a 'HST-sTIS eclipse': maybe occultation is a better word, though what we appear to be seeing in the X-rays is an 'elipse' of the X-ray emitting region by (presumably) the wind from the front-most star. Right now, the flare we saw in April 2002 is a puzzle. From the X-ray spectra it appears to be a change in emission measure, suggesting an increased density in the wind near the colliding wind shock. It would be very interesting, though, if the real X-ray period is not the same as the He I $10830 \AA$ line period. That could mean that the X-rays (perhaps) are less affected by nebular variability - or maybe, that the colliding wind binary scenario is wrong.

WILLIS: Magnesium is an element which may show non-solar abundances in WC stars, via nuclear processing in He-burning and $\alpha$-capture. There are no good $\mathrm{Mg}$ diagnostics in optical/UV/IR spectra, yet there are good Mg lines in soft X-ray spectra, that you have from Chandra and XMM-Newton. Is anyone determining $\mathrm{Mg}$ abundances in WC CWB systems, from these X-ray spectra?

CoRCORAN: We are attempting this for WR 140. Using simple collisional plasma models doesn't show evidence for large enhancements in magnesium (if I remember correctly), but I think we need to do a detailed hydro-model to get a better hand on it.

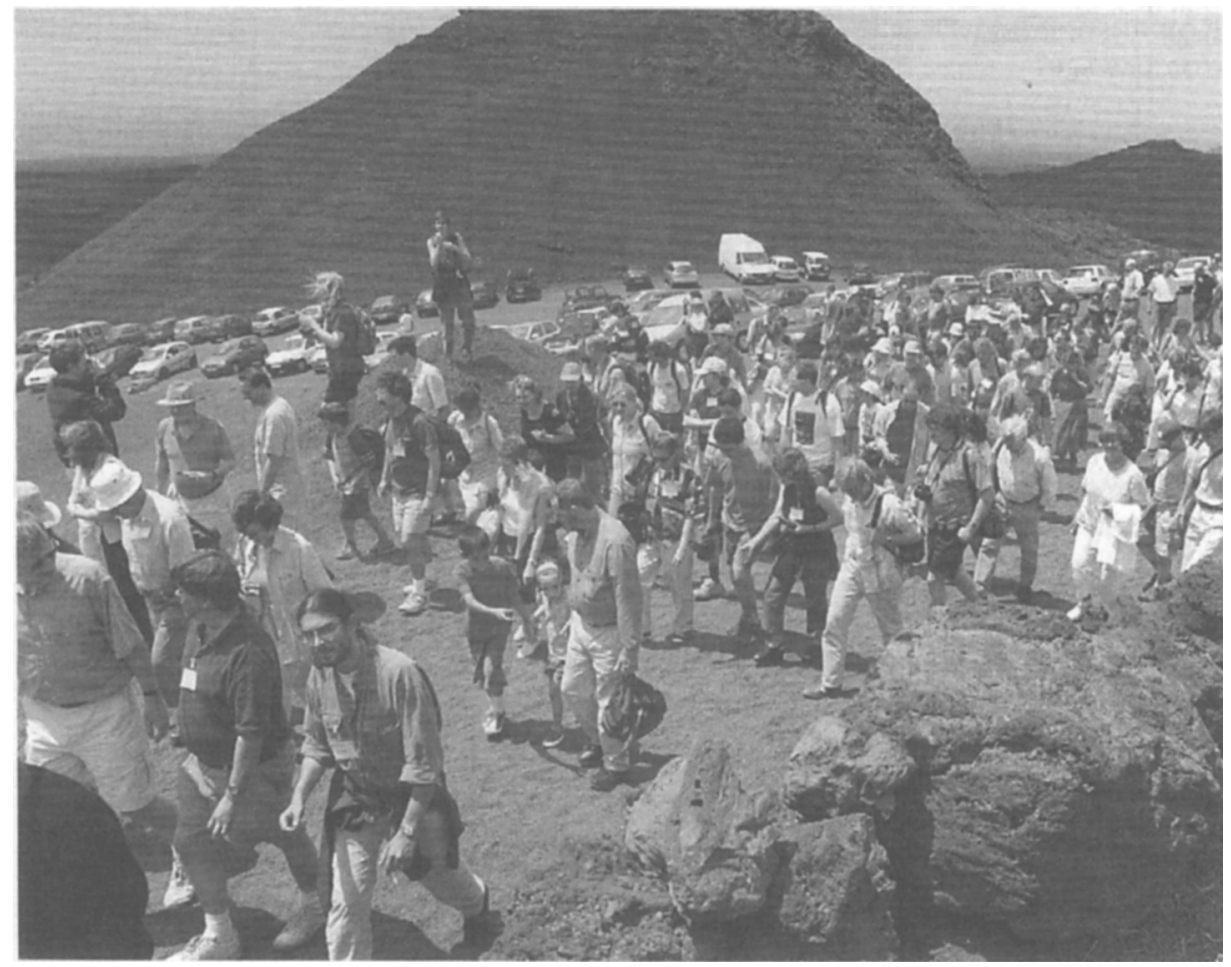

Lex Kaper, Mike Corcoran and Olivier Schnurr, leading the crowd in Timanfaya National Park 